\title{
Das „Sekretärchen“ und der „Künstler im ächten Sinne des Wort's“
}

\author{
Kollektives Schreiben unter dem Aspekt der Geschlechtlichkeit
}

\author{
Jennifer Clare
}

Im Jahr 1991 schreibt Barbara Hahn über, wie es im Untertitel ihres Buches heißt, die schwierige Autorschaft der Frauen:

Der Gegenpol zum modernen Autor mit dem einen Namen, der ein Werk zusammenhält und regiert, ist eine unbezeichenbare Vielheit von Texten, die unautorisiert und zwischen den Genres wandernd immer wieder aus dem Kanon des Überlieferbaren ausscheren: Was nicht benannt werden kann, läßt sich schwer tradieren. ${ }^{1}$

Der einzelne Name als Klammer für ein Werk, als Chiffre für einen Vorgang der Textproduktion, ist die gängigste und sichtbarste Form der Abbildung und Speicherung von Autorschaft - und zugleich eine Form, die, wie Hahn zeigt, vor allem im 18. und 19. Jahrhundert auf die Schreib- und Publikationspraxis männlicher Schreibender zugeschnitten ist und weibliche Schreibende durchs Raster fallen lässt. Die Eigenschaften, die Hahn hier für weibliches Schreiben festhält, lassen sich fast nahtlos auf den Fall kollektiven Schreibens übertragen: Auch kollektives Schreiben entzieht sich häufig der Repräsentation über einen einzelnen Namen, ein geschlossenes Werk und schert damit gegebenenfalls aus Dynamiken von Genre und Kanon aus. Was bedeutet diese Gemeinsamkeit für die wissenschaftliche Annäherung an kollektives Schreiben? Anders gefragt: Wenn die singuläre, durch nur einen Namen repräsentierte Autorschaft ein potenziell geschlechtlich codiertes Phänomen ist, gilt das auch für ihren ,Gegenpart', die Autorschaft mehrerer Personen an einem Text?

Eine systematische wissenschaftliche Auseinandersetzung mit den geschlechtlichen Aspekten kollektiven Schreibens steht bislang aus. Der vorliegende Beitrag soll erste Denkansätze liefern und kann dabei auf zahlreiche Forschungsergebnisse benachbarter Themengebiete aufbauen: Es gibt seit den 1970er Jahren eine ausgesprochen reiche deutsch- und englischsprachige

1 Barbara Hahn, Unter falschem Namen. Von der schwierigen Autorschaft der Frauen, Frankfurt/ Main: Suhrkamp 1991, S. 7. 
Forschung zu weiblicher Autorschaft ${ }^{2}$ sowie eine große Zahl von Einzelstudien zu gemeinsam schreibenden Männern und Frauen. Letztere reichen von Fällen gemeinsam schreibender Paare ${ }^{3}$ über Konstellationen nur zwischen Frauen ${ }^{4}$ oder nur zwischen Männern ${ }^{5}$ bis hin zur Frage nach der Schreibumgebung als Ort praktizierter und verhandelter Geschlechtlichkeit. ${ }^{6}$ Parallel dazu haben sich in den letzten Jahren in der neueren Autorschaftsforschung Ansätze entwickelt, die das Konzept der Autorschaft generell von der stiftführenden und namentlich genannten Person zu entkoppeln suchen und die für die Frage nach kollektivem Schreiben gleichermaßen relevant sind (siehe Kapitel 1.1).

In einem ersten Schritt soll dieses Material daraufhin gesichtet werden, wie Schreibprozess und Autorschaft überhaupt „kollektiv“ denkbar sind, mit welchen Begriffen und theoretischen Prämissen bislang operiert wird und wie sie jeweils zu einer singulären Autorschaft ins Verhältnis gesetzt werden. In einem zweiten Schritt soll der Blick auf die geschlechtlichen Implikationen dieser Überlegungen gerichtet werden. Welche Fragen tun sich auf zwischen Kollektiv, Schreiben und Geschlecht? Ist Geschlechtlichkeit überhaupt ein relevanter Aspekt für die Beschreibung eines kollektiven Schreibens? Und können Beschreibungsmuster erweiterter Autorschaft und geschlechtlich

2 Angefangen von "Klassikern“ der feministischen Literaturwissenschaft aus den 1970er Jahren (exemplarisch genannt seien Silvia Bovenschen, Die imaginierte Weiblichkeit. Exemplarische Studien zu kulturgeschichtlichen und literarischen Präsentationsformen des Weiblichen, Frankfurt/Main: Suhrkamp 1979, sowie Sandra M. Gilbert und Susan Gubar, The Madwoman in the Attic: The Woman Writer and the Nineteenth-Century Literary Imagination, New Haven, London: Yale University Press 1979) über epochenübergreifende Übersichten weiblicher $\mathrm{Au}-$ torschaft (etwa Barbara Becker-Cantarino, Der lange Weg zur Mündigkeit. Frauen und Literatur in Deutschland von 1500 bis 1800, München: dtv 1989; Hiltrud Gnüg und Renate Möhrmann (Hrsg.), Frauen Literatur Geschichte. Schreibende Frauen vom Mittelalter bis zur Gegenwart, Stuttgart: Metzler 1985; Gisela Brinker-Gabler (Hrsg.), Deutsche Literatur von Frauen, 2 Bde., München: Beck 1988) bis hin zu Studien zu speziellen Konstellationen und einzelnen Autorinnen (etwa Birgit Schütte, Weibliche Perspektiven in der Gegenwartsliteratur, Frankfurt/Main u.a.: Lang 1999).

3 Aktuell beispielsweise Ingrid Bauer und Christa Hämmerle (Hrsg.), Liebe schreiben. Paarkorrespondenzen im Kontext des 19. und 20. Jahrhunderts, Göttingen: Vandenhoeck \& Ruprecht 2017, und Tania Schlie, Schreibende Paare. Liebe \& Literatur, München: Thiele 2016.

4 Beispielsweise Bette London, Writing Double. Women's literary partnerships, Ithaka, London: Cornell University Press 1999 .

5 Beispielsweise Wayne Koestenbaum, Double Talk: The Erotics of Male Literary Collaboration, New York: Routledge 1989.

6 Beispielsweise Lorraine York, „Crowding the Garret. Women's Collaborative Writing and the Problematics of Space“, in: Marjorie Stone und Judith Thompson (Hrsg.), Literary Couplings. Writing Couples, Collaborators, and the Construction of Authorship, Madison: University of Wisconsin Press 2006, S. 288-307. 
markierter Autorschaft produktiv zusammengeführt werden? In einem dritten Schritt werden diese Fragen anhand von ausgewählten Schreibprojekten des 19. Jahrhunderts exemplarisch diskutiert.

\section{Kollektive Implikationen von Schreibprozess und Autorschaft}

\subsection{Kollektives Schreiben, kollaboratives Schreiben und multiple authorship - über Begriffe und ihre Voraussetzungen}

Für den Fall eines Schreibprozesses, an dem mehr als eine Person beteiligt ist, sind zahlreiche verwandte Bezeichnungen im Umlauf. Sie sind allerdings, wie Claas Morgenroth anhand der Begriffe „kollaboratives“ und „kollektives Schreiben" zeigt, von ihrer Wortherkunft und auch literaturwissenschaftlichen Ausrichtung her nicht immer deckungsgleich. Betont beispielsweise kollaboratives Schreiben die gemeinsam erledigte Arbeit im Schreiben, ist kollektives Schreiben ein deutlich weiter gefasster Begriff, der innerhalb der Literaturgeschichte eine starke Verwendungstradition im Kontext politisierten Schreibens hat. ${ }^{7}$ Morgenroths Unterscheidung erlaubt, eine erste Schneise in die Begrifflichkeiten und ihre Schwerpunkte zu schlagen: Es gibt Begriffe, die sich auf konkrete Personen beziehen, die Aufgaben im Rahmen eines Schreibprozesses übernehmen. „Kollaboratives Schreiben“ gehört dazu, ebenso wie „literary couplings" ${ }^{\prime}$, "literary partnerships“, 9 "joint creation“, ${ }^{10}$ „,silent authorship“11 und „literarische Zusammenarbeit". ${ }^{12}$ Wie man sieht, stellen manche Bezeichnungen die schreibenden Personen in den Vordergrund, andere den Prozess - aber gemeinsam ist ihnen, dass sie von klar benennbaren Beteiligten an einem konkreten Arbeitsprozess ausgehen, die in einer persönlichen Beziehung zueinander stehen. Auf der anderen Seite stehen Ansätze, die sich unter verschiedenen theoretischen Voraussetzungen mit Schreibprozess und Autorschaft im größeren soziokulturellen Zusammenhang befassen.

Vgl. Claas Morgenroth, „Kollaboratives Arbeiten und kollektives Schreiben“, in: Undercurrents - Forum für linke Literaturwissenschaft 7 (2016), http://undercurrentsforum. com/2016/o1/o7/ (letzter Zugriff: 24.10.2018), S. 2.

8 Stone/Thompson, Literary Couplings (Anm. 6).

$9 \quad$ London, Writing Double (Anm. 4).

10 Thomas Hines, Collaborative Form. Studies in the Relations of the Arts, Kent/Ohio: Kent State University Press 1991, S. 4.

11 Ruth Perry und Martine Watson Brownley (Hrsg.), Mothering the Mind. Twelve Studies of Writers and Their Silent Partners, New York: Holmes \& Meyer 1984.

12 Bodo Plachta (Hrsg.), Literarische Zusammenarbeit, Tübingen: Niemeyer 2001. 
Einer der ersten Ansätze dieser Art stammt von dem Anglisten Jack Stillinger. Stillingers Studie Multiple Authorship and the Myth of Solitary Genius zeigt in stark kanonisierten Texten der englischen Literaturgeschichte umfassende Arbeitsprozesse, die sich der Repräsentation durch den einzelnen, bekannten Namen (John Keats, William Blake) entziehen. Dieses Zusammenwirken der kanonisierten Autorinstanz mit anderen mitschreibenden Instanzen nennt Stillinger "multiple authorship“. ${ }^{13}$ Sie ist ein frühes Beispiel für den Versuch, das Konzept von Autorschaft über die Einzelperson hinaus zu erweitern: Stillinger versucht einerseits, die stumme Mitverfasserschaft von Angehörigen des professionellen oder privaten Umfelds der schreibenden Person abzubilden. Andererseits legt er den Fokus darauf, in welche Netzwerke, Kommunikationszusammenhänge und auch Machtgefüge des Literaturbetriebs die schreibende Person und ihr Text eingebunden sind. Dieses abstraktere Verständnis von Kollektivierung im Schreiben greifen die von Gerhard Fischer und Florian Vaßen untersuchte "collective creativity“, ${ }^{14}$ die aktuell untersuchten Fälle „relationaler Autorschaft" ${ }^{\text {"15 }}$ bzw. „literarischer Netzwerke“16 sowie frühere Studien zu Autorschaft und Literaturbetrieb wie die von Stephan Porombka ${ }^{17}$ auf. All diesen (in ihren literaturtheoretischen Grundlagen durchaus heterogenen) Ansätzen ist gemeinsam, dass es ihnen weniger auf das Zusammenwirken bestimmter schreibender Personen ankommt als auf die Einbindung von einzelnen Autorinnen und Autoren und ihren Schreibprozessen in zum Beispiel soziale, politische und kulturelle Kollektive.

13 Vgl. Jack Stillinger, Multiple Authorship and the Myth of Solitary Genius, New York: Oxford University Press 1991, S. V. Der Begriff der Autorschaft ist mittlerweile gegenüber anderen Schreibpositionen präzisiert worden, vor allem durch Christoph Hoffmann. Nach Hoffmanns Unterscheidung der Schreibpositionen "Schreiber“, "Verfasser“ und "Autor" wird nicht die Autorschaft der von Stillinger untersuchten Texte geteilt (insofern die Foucault'sche Autorfunktion weiterhin nur für Keats bzw. Blake nachweisbar ist), sondern die Verfasserschaft (vgl. dazu Christoph Hoffmann, „Schreiber, Verfasser, Autoren“, in: Deutsche Vierteljahrsschrift für Literaturwissenschaft und Geistesgeschichte 91 (2017), H. 2, S. 163-187).

14 Gerhard Fischer und Florian Vaßen (Hrsg.), Collective Creativity. Collaborative Work in the Sciences, Literature and the Arts, Amsterdam, New York: Rodopi 2011.

15 Hannes Fischer und Daniel Zimmer, „Werke im Netzwerk. Relationale Autorschaft im 18. Jahrhundert (Workshop in Berlin v. 11.-12.5.2017)“. Konferenzbericht, in: Zeitschrift für Germanistik 28 (2018), H. 1, S. 137-140.

16 Hannes Fischer und Erika Thomalla, „Literaturwissenschaftliche Netzwerkforschung zum 18. Jahrhundert“, in: Zeitschrift für Germanistik 26 (2016), H. 1, S. 110-117, hier S. 111.

17 Stephan Porombka, „Literaturbetriebskunde. Zur ,genetischen Kritik kollektiver Kreativität“, in: ders. (Hrsg.), Kollektive Kreativität, Tübingen: Francke 20o6, S. 71-86. 
Es wird deutlich, dass die skizzierten Ansätze sehr unterschiedliche Phänomene meinen, wenn sie davon sprechen, dass an einem Schreibprozess mehrere Personen beteiligt sind: Sie reichen von der engen, räumlich und persönlich stark verbundenen Zusammenarbeit zweier oder dreier benennbarer schreibender Personen bis zu den Auswirkungen, welche das einen Schreibprozess umgebende soziale Kollektiv auf den entstehenden Text hat.

Der vielleicht flexibelste Begriff in diesem Diskursgefüge ist das „kollektive Schreiben“, das dem vorliegenden Band den Titel gibt. Er ist sowohl für Fragen nach schreibender Zusammenarbeit von konkreten Personen gebräuchlich als auch für Fragen nach dem Wirken von größeren Kollektiven auf Schreibprozess und Text, kann also sowohl die Mikro- als auch die Makroperspektive ausdrücken. Aus diesem Grund wird er im Folgenden in diesem Rahmen als Oberbegriff für alle Arten von Schreibprozessen mit mehreren Beteiligten verwendet.

\subsection{Fragestellungen und Themenbereiche kollektiven Schreibens}

Der kleine Überblick über die bestehenden Begrifflichkeiten eröffnet bereits eine Reihe von verfolgenswerten Anknüpfungspunkten zur Beschreibung kollektiver Schreibprozesse. Ein erster wichtiger Aspekt scheint, wie viele Beteiligte im Spiel sind. Diese scheinbar banale Frage entscheidet, wie oben skizziert, tendenziell über die theoretische und gegenständliche Ausrichtung: Werden eher Personen in ihrer Zusammenarbeit und ihren Beziehungen oder größere soziale Dynamiken im Rahmen von Autorschaft untersucht? Eng daran gekoppelt ist die Frage danach, auf welcher Textgrundlage von einem kollektiven Schreiben gesprochen wird: Ist das Ergebnis ein Text (z.B. ein gemeinsam geschriebener Roman) oder eine Gruppe aufeinander bezogener Texte (z.B. ein Briefwechsel, ein Zeitschriftenprojekt) oder ein über viele Texte zusammenhängendes und realisiertes soziales Gefüge?

Wie die Konzepte von multipler, stummer oder relationaler Autorschaft bzw. Verfasserschaft zeigen, ist darüber hinaus der Aspekt der öffentlichen Repräsentation von Relevanz: Wird das Ergebnis des kollektiven Schreibens überhaupt publiziert, und wenn ja, durch welche(n) Namen wird es ausgewiesen? Vor allem Fälle stummer Verfasserschaft - also Fälle, in denen eine Person maßgeblich am Schreibprozess beteiligt ist, aber nicht namentlich erscheint - zeigen, dass die praktische Umsetzung eines Schreibprozesses und seine öffentliche Abbildung stark auseinandergehen können. Von daher ist die Untersuchung von kollektivem Schreiben auf die Untersuchung von Schreibprozessen angewiesen, insofern man davon ausgehen muss, dass der publizierte Text nicht immer alle Beteiligten benennt, die potenziell von Interesse sind. 
Für die Untersuchung von Schreibprozessen stehen wertvolle methodische Vorarbeiten aus der Schreibforschung zur Verfügung, von der critique génétique ${ }^{18}$ bis $\mathrm{zu}$,genealogischen' Ansätzen wie der Schreibszene. ${ }^{19}$ Zwar legen die meisten dieser Ansätze die Prämisse einer singulären Autorschaft zugrunde, sie lassen sich jedoch, wie ich an anderer Stelle gezeigt habe, ${ }^{20}$ durchaus um Aspekte des Kollektiven erweitern. Eine Konsequenz ist allerdings, dass der Begriff des Schreibens dabei grundsätzlich auf den Prüfstand gelangt. Solange sich die Frage nach dem Schreiben um eine Person zentriert, ist der Fokus durch ihre Handlungen am Text und den Kontext dieser Handlungen recht klar bestimmt. Treten jedoch weitere Akteurinnen und Akteure hinzu, kommt unweigerlich die Frage auf, welche und wessen Handlungen überhaupt zum Schreibprozess gehören. Gerade im Bereich abstrakter sozialer Zusammenhänge gehen die denkbaren Handlungsbezüge gegen unendlich, doch schon bei zwei oder drei Schreibenden ist die Entscheidung, ob eine Handlung zu einer Veränderung im Text geführt hat, selten absolut zu beantworten. ${ }^{21}$ Eine Schreibforschung, die sich kollektivem Schreiben zuwendet, ist darauf angewiesen, jeden Einzelfall in dieser Hinsicht individuell zu bewerten. Ein wichtiger Ausgangspunkt dafür kann etwa

18 Vgl. einführend Louis Hay, „Die dritte Dimension der Literatur. Notizen zu einer critique génétique (1984), in: Sandro Zanetti (Hrsg.), Schreiben als Kulturtechnik. Grundlagentexte, Frankfurt/Main: Suhrkamp 2012, S. 132-151, und Almuth Grésillon, „Was ist Textgenetik?“, in: Jürgen Baurmann und Rüdiger Weingarten (Hrsg.), Schreiben. Prozesse, Prozeduren und Produkte, Opladen: Westdeutscher Verlag 1995, S. 288-319.

19 Vgl. einführend Rüdiger Campe, „Die Schreibszene, Schreiben“ (1991), in: Zanetti (Hrsg.), Schreiben als Kulturtechnik (Anm. 18), S. 269-282, sowie Martin Stingelin, „Schreiben“. Einleitung“, in: ders. (Hrsg.), „Mir ekelt vor diesem tintenklecksenden Säkulum“. Schreibszenen im Zeitalter der Manuskripte, München: Fink 2004, S. 7-21.

20 Vgl. Jennifer Clare, „Schrift als Dokument und Praxis literarischer Zusammenarbeit“, in: Monika Schmitz-Emans, Linda Simonis und Simone Sauer-Kretschmer (Hrsg.), Schrift und Graphisches im Vergleich, Bielefeld: Aisthesis 2019, S. 469-481.

21 Welche Auswirkungen enge und weite Schreibbegriffe auf die Wahrnehmung von Schreibprozessen als singulär oder kollektiv haben können, zeigt die interessante Studie von Lisa Ede und Andrea Lunsford zu kollektiven Schreibprozessen im professionellen Umfeld: „An early question asked what percentage of time these professionals spent writing alone and what percentage writing as a team or with a group: they reported writing alone 82 percent of the time. The wording of the question and the commonsense view of writing as the physical act of putting pen or typewriter key to paper probably contributed to this anomalous response, for later answers contradicted this one" (Lisa Ede und Andrea Lunsford, Singular Texts/Plural Authors: Perspectives on Collaborative Writing, Carbondale: South Illinois University Press 199o, S. 6o). Mit einem engen Schreibbegriff („putting pen or typewriter key to paper“) werden viele Aktivitäten rund um den entstehenden Text von den Probandinnen und Probanden entweder nicht als gemeinsam oder nicht als Teil des Schreibens wahrgenommen. 
sein, wie sich schreibende Personen im Rahmen von Schreib-Szenen ${ }^{22}$ zu anderen Beteiligten und deren Handlungen positionieren oder wessen Handlungen Spuren am Material hinterlassen haben (in Kapitel 3 werden Beispiele für beides zu sehen sein). Diese Einsichten in Schreibprozesse sind wiederum interessant in Relation zur bereits erwähnten Frage der öffentlichen Repräsentation ihrer Ergebnisse: Welche Handlungen sind es, die am Ende zur Zuschreibung „Autor“ bzw. „Autorin“ führen, und welche werden in der öffentlichen Wahrnehmung in die Ränge von Zuarbeit, Unterstützung und Einfluss verwiesen oder gänzlich marginalisiert? Howard Becker hat gezeigt, dass in modernen künstlerischen Arbeitsprozessen in der Regel zwischen künstlerischen und nicht-künstlerischen Tätigkeiten unterschieden wird:

\begin{abstract}
Einige der Tätigkeiten, die notwendig sind für die Produktion eines Kunstwerkes, werden von den an einer Kunstwelt Beteiligten als ,künstlerische‘ Tätigkeiten erachtet. Diese bedürfen eines speziellen Talents oder einer Sensibilität des Künstlers. Die übrigen Tätigkeiten erscheinen ihnen als eine Sache des Handwerks, der Geschäftstüchtigkeit oder einer Fähigkeit geschuldet, die weniger selten, weniger charakteristisch für Kunst, weniger notwendig für den Erfolg einer Arbeit und weniger anerkannt ist. Personen, die die beschriebenen spezifischen Tätigkeiten ausführen, definieren sie als Künstler und alle anderen als unterstützendes Personal. ${ }^{23}$
\end{abstract}

Demnach dominiert in der Produktion und Rezeption von Kunstwerken die Vorstellung einiger wesentlicher und unersetzbarer Tätigkeiten, die besonders hohe Anerkennung verdienen und das Recht verleihen, das Kunstwerk vor dem Publikum (und oft eben auch der Forschung) namentlich allein zu repräsentieren. Bei der Erforschung kollektiver Schreibprozesse ist also auch die konkrete Organisation des Schreibprozesses ein wichtiger Aspekt und, eng verbunden, das spezifische Verhältnis von Produktion und Rezeption. Lässt sich etwas sagen über quantitative und qualitative Anteile der Beteiligten an einem Schreibprozess? Wer stößt ihn an, wer plant ihn? Gibt es eine klare Arbeitsteilung? Werden Entscheidungen gemeinsam getroffen oder gibt es eine Person mit Entscheidungshoheit? Dieser Bereich ist, wie in Bezug auf den Aspekt der Geschlechtlichkeit zu sehen sein wird, nicht ganz unproblematisch,

22 Eine Schreib-Szene liegt nach Martin Stingelin vor, wenn Elemente aus der Schreibszene eines Textes im Text selbst thematisiert und reflektiert werden, d.h. sich in ihren Eigenheiten und gegebenenfalls auch als Störfaktoren unmittelbar textkonstitutiv auswirken (vgl. Stingelin, „Schreiben'. Einleitung“ [Anm. 23], S. 15).

23 Howard S. Becker, „Kunst als kollektives Handeln“, in: Jürgen Gerhards (Hrsg.), Soziologie der Kunst. Produzenten, Vermittler und Rezipienten, Opladen: Westdeutscher Verlag 1997, S. 23-40, hier S. 25 . 
insofern er die schwierige Aufgabe auferlegt, Leistungen zu bewerten - unter Umständen auf Basis subjektiver Selbstdarstellungen. Ich werde darauf zurückkommen.

Einen näheren Blick verdient in vielen Fällen auch das Verhältnis zwischen produzierender und rezipierender Instanz. Thomas Ernst hat im Anschluss an Hajo Kurzenberger gezeigt, "that an admiring mass or collective has always been the necessary opposite to every individual author-genius“. ${ }^{24}$ Die mächtigste Konstellation der Neuzeit ist die des produzierenden, singulären Originalgenies, das einer anonymen, rezipierenden Leserschaft gegenübersteht. Im Fall eines kollektiven Schreibens sieht das Verhältnis jedoch unter Umständen ganz anders aus: Schon der geteilte Schreibprozess zweier Personen an einem Text ist notwendigerweise einer, in dem sich Schreiben und gegenseitiges Lesen, Produktion und Rezeption, stetig abwechseln. ${ }^{25}$

Bevor die herausgearbeiteten Aspekte kollektiven Schreibens auf ihre geschlechtlichen Implikationen befragt werden, ist ein kurzer Blick darauf notwendig, wie unterschiedlich die einzelnen Ansätze das Verhältnis zwischen kollektivem Schreiben und Autorschaft fassen. Wie Marjorie Stone und Judith Thompson betonen, ist die singuläre Autorinstanz in fast jeder literaturwissenschaftlichen Praxis einer der wichtigsten Bezugspunkte: „[T]he author classification remains the dominant means of codifying, archiving, teaching, analyzing, and legislating literary works.“26 Diese mächtige Struktur führt vor allem in älteren Auseinandersetzungen mit gemeinsam geschriebenen Texten zu der eher unproduktiven Lösung, dass die beteiligten Schreibenden einfach

24 Thomas Ernst, „From Avant-Garde to Capitalistic Teamwork: Collective Writing Between Subversion and Submission“, in: Fischer/Vaßen (Hrsg.), Collective Creativity (Anm. 14), S. 229-242, hier S. 232 f.

25 Sehr gut zu sehen etwa in Heinz Rölleckes Analysen der gemeinsamen Schreibprozesse von Achim von Arnim und Clemens Brentano sowie Wilhelm und Jacob Grimm. Vgl. Heinz Röllecke, „,Wie die Dioskuren“ - Art und Ergebnisse literarischen Zusammenwirkens in der Romantik“, in: Plachta (Hrsg.), Literarische Zusammenarbeit (Anm. 12), S. 131-140. Noch extremer kippt das Verhältnis in sogenannten partizipativen Formaten Formaten also, in denen die Rezipientenschaft als aktiver Teil im Entstehungsprozess des Textes mitgedacht wird. Zu solchen Schreibformaten zu unterschiedlichen Zeiten und in unterschiedlichen Medien vgl. den aktuellen Band von Martin Butler, Albrecht Hausmann und Anton Kirchhofer (Hrsg.), Precarious Alliances. Cultures of Participation in Print and Other Media, Bielefeld: transcript 2016.

26 Stone/Thompson (Hrsg.), Literary Couplings (Anm. 6), S. 12; vgl. ähnlich auch Stillinger, Multiple Authorship (Anm. 13), S. VI: „Most theories of interpretation and editing are based on the idea of a single author [...] as sole controlling intelligence in a work. We routinely refer to a single authorial mind, or personality, or consciousness to validate ,meaning' or ,authority'; where others besides the nominal author have a share, we usually ignore that share or else call it corruption and try to get rid of it.“ 
wie zwei oder drei singuläre Autorinnen und Autoren behandelt werden, die im Text zusammentreffen und deren Anteile es möglichst präzise zuzuweisen und zu separieren gilt. Kollektives Schreiben erscheint in dieser Logik als die summierten Tätigkeiten mehrerer singulärer Autorschaften. ${ }^{27}$ Ein solches Vorgehen verkennt aber erstens die komplexen Verflechtungen aus Produktion und Rezeption, die kollektive Schreibprozesse prägen, und erweist sich zweitens als unsensibel dafür, dass kollektives Schreiben in ganz unterschiedliche Repräsentationen münden kann.

Auf der anderen Seite steht das Extrem, die Existenz von singulärer Autorschaft per se zu negieren. Bei Jack Stillinger etwa ist das kollektive Schreiben kein seltenes Abweichen vom Allein-Schreiben, sondern mehr oder weniger stark ausgeprägte Grundlage jeglicher Textproduktion. Stillinger kritisiert das Konzept der singulären Autorschaft als überholtes und unreflektiertes Erbe der Genieästhetik und zeigt anhand der sehr stark als ,Originalgenies‘ kanonisierten Autoren Keats und Blake, wie auch deren Texte sowohl von anderen Personen als auch von Dynamiken des Literaturbetriebs und ", historical and cultural circumstances“28 bearbeitet werden. Einen ähnlichen Weg gehen Stone und Thompson, wenn sie vorschlagen, jegliche Autorschaft weniger von einem geschlossenen Personenleben aus zu denken denn als komplexen Kondensationspunkt verschiedener Texte, Stimmen und Handlungen:

We propose that the paradigm of the unitary author, which has thus far proven to be more resistant to transformation than the idea of the unitary text, should similarly be replaced by a conception of authors as ,heterotexts', woven of varying strands of influence and agency, absorbing or incorporating differing subjectivities, and speaking in multiple voices. ${ }^{29}$

Der Reiz an Ansätzen wie diesen, die die kollektive Natur jeglichen Schreibprozesses betonen, liegt darin, Texte und Schreibprozesse kausal von einzelnen Personen abzukoppeln. Sie befreien vom Zwang, Texte in namentlich

27 Wie wenig angemessen diese Herangehensweise ist, führt Bette London aus: „The questions literary collaborators reported as most frequent and persistent - Which hand held pen? (or, put another way, which is the real author? Who did the actual writing?) were, by general consensus, not very productive. Nor were the equally pervasive but more specific attempts to pin down the collaborators' precise division of labor. As Susan Leonardi remarks, ,Anyone who has ever written, or even cooked, with someone else knows that this is not how it works' - an insight confirmed by other scholars and practitioners of collaboration" (London, Writing Double [Anm. 4], S. 25; zit. n. Susan Leonardi und Rebecca Pope, „Screaming Divas: Collaboration as Feminist Practice“, in: Tulsa Studies in Women's Literature 13 (1994), H. 2, S. 259-270, hier S. 259).

28 Stillinger, Multiple Authorship (Anm. 13), S. 185.

29 Stone/Thompson (Hrsg.), Literary Couplings (Anm. 6), S. 19. 
benennbare Einzelleistungen zu zergliedern - was für die Betrachtung komplexer Schreibkonstellationen sehr hilfreich sein kann. Sie bergen aber zugleich die Gefahr, jegliche Grenzen zu verwischen zwischen der generellen sozialen Einbettung, die jeder Text besitzt, und der prominenten kollaborativen Dimension, die nur manche Texte haben. Die Einsicht, dass jeder Text sozial eingebettet ist, entlastet also nicht vom differenzierten Blick auf den Individualfall, auf dessen genaue Konstellation von Akteurinnen und Akteuren und auf die Bedeutung des kollektiven Anteils für den resultierenden Text.

In jedem Fall bedenkenswert ist die These von Stone und Thompson, wonach die singuläre Autorschaft durchaus existiert - aber resultierend aus einem nachträglichen Prozess der Inszenierung und der Homogenisierung. Sie wirft die berechtigte Frage auf, ob sich dem Phänomen des kollektiven Schreibens die Kontrastfolie eines singulären Schreibens überhaupt sinnvoll gegenüberstellen lässt, wenn singuläre Autorschaft kein tatsächlicher Ausgangspunkt für Schreibprozesse ist, sondern etwas, das dem Text im Rahmen von gesellschaftlichen, wirtschaftlichen und literaturbetrieblichen Dynamiken nachträglich zugeschrieben wird. Thomas Ernst schlägt vor, „to distinguish between the real process of creativity and production [...] and the staged performance of authorship on the judicial and economic fields of society. ${ }^{40}$ Die singuläre Autorschaft findet dann ihren Ort als zu analysierende Ausprägungsform der öffentlichen Inszenierung eines Schreibprozesses. Diese Differenzierung ist für das folgende Verständnis von kollektivem Schreiben wichtig: Es gibt kein nicht-kollektives Schreiben, aber sowohl die Dimensionen und Grade der Arbeitsteilung und des Austauschs als auch die Inszenierung seiner Ergebnisse fallen sehr unterschiedlich aus - bis dahin, dass sie in der Herstellung einer singulären Autorschaft aufgehen. All diese Dynamiken wollen im Einzelfall genau untersucht werden - gerade wenn es wie im Folgenden darum geht, geschlechtlich codierte Elemente in diesem Schreibprozessen zu erfassen.

\section{Geschlechtliche Implikationen von Schreibprozess und Autorschaft}

Bevor es um die geschlechtlichen Implikationen der zuvor vorgestellten Forschungsperspektiven zum kollektiven Schreiben geht, stellt sich die Frage, was es grundsätzlich heißt, Schreibprozess und Autorschaft in Bezug auf Geschlechtlichkeit zu untersuchen. Zunächst bedeutet es, zwischen Schreiben, Autorschaft und Geschlecht unterschiedliche Berührungspunkte

30 Ernst, „From Avant-Garde to Capitalistic Teamwork“ (Anm. 24), S. 234. 
wahrzunehmen: Wir haben es mit geschlechtlich markierten Körpern zu tun, die Schreibhandlungen durchführen. Wir haben es mit männlichen und weiblichen Namen zu tun, unter denen Texte erscheinen, sowie mit geschlechtlicher Performanz im Rahmen eines Schreibprozesses. Diese Elemente können sich innerhalb eines Schreibprozesses decken, müssen es aber nicht: Zum Beispiel publizieren viele Frauen im 19. Jahrhundert unter männlichen Pseudonymen und agieren unter Umständen auch sozial als Männer, etwa im Kontakt mit Instanzen des Literaturbetriebs oder um ungestört reisen zu können. ${ }^{31}$ Gerade die geschlechtliche Performanz im Schreibprozess muss gänzlich unabhängig vom biologischen Körper betrachtet werden, denn es ist ohne Weiteres denkbar, dass ein schreibender Mann weiblich codierte Sprach- und Verhaltensmuster übernimmt oder umgekehrt. Während der schreibende Körper in aller Regel in seiner Geschlechtlichkeit konstant bleibt, kann sich der Name, der die Schreibprozesse dieses Körpers vertritt, mehrfach ändern. ${ }^{32}$ Die geschlechtliche Performanz ist noch flexibler und steht mit dem Schreibprozess in einer Wechselbeziehung: Jedem Schreibprozess - wie übrigens jedem Leseprozess auch - gehen eine geschlechtliche Sozialisation, historische und kulturelle Voraussetzungen der Geschlechtlichkeit, voran, aber sie werden in ihm auch verhandelt, in Szene gesetzt, affirmiert oder unterlaufen. ${ }^{33}$ Eine Untersuchung von Schreiben und Geschlecht muss all diese Berührungspunkte im Blick behalten.

Ich möchte im Folgenden mit einigen Beispielen aus dem 19. Jahrhundert zeigen, wie Schreiben, Autorschaft und Geschlecht aufeinander bezogen sein können und wie dies für das kollektive Schreiben auswertbar ist. Das 19. Jahrhundert ist keine ganz beliebige Wahl: Die Jahrhundertwende um 1800 markiert sowohl für den Umgang mit Geschlechtlichkeit als auch mit dem

31 Susanne Kord, Sich einen Namen machen. Anonymität und weibliche Autorschaft 1700-1900, Stuttgart: Metzler 1996, S. 54 und S. 181-201, listet für das 19. Jahrhundert 476 Beispiele für bekannte männliche Pseudonyme von Autorinnen auf. Zur weiblichen Reisepraxis und -literatur vgl. Annegret Pelz: ,.... von einer Fremde in die andre?' Reiseliteratur von Frauen“, in: Brinker-Gabler (Hrsg.), Deutsche Literatur (Anm. 2), S. 143-153.

32 Barbara Hahn führt für das 19. Jahrhundert u.a. die Beispiele der gebürtigen Brendel Mendelssohn und Esther Gad an, die im Laufe ihres Lebens durch Eheschließungen oder christliche Taufe unter verschiedenen Namen (Brendel Veit, Dorothea Veit, Dorothea Schlegel/Esther Bernard, Lucie Domeier) schreibend in Erscheinung getreten sind, und thematisiert in diesem Zusammenhang ausführlich, wie Geschlecht, Familienstand und Religion sich auf die Anerkennung und auch das Selbstbild als Autorin auswirken (Hahn, Unter falschem Namen [Anm. 1], S. 21ff. und S. 43ff.). Vgl. zur geschlechtlichen Dimension des Autornamens auch Kord, Sich einen Namen machen (Anm. 31).

33 Vgl. Sigrid Nieberle, Gender Studies und Literatur. Eine Einführung, Darmstadt: Wissenschaftliche Buchgesellschaft 2013, S. 68-76. 
Schreiben eine wichtige Zäsur. In Friedrich Kittlers Worten wird das 19. Jahrhundert gerahmt von zwei einflussreichen „Aufschreibesystemen“, mit denen die medialen und sozialen Voraussetzungen des Schreibens grundlegend neu gesetzt und verhandelt werden. ${ }^{34}$ Vom ausgehenden 18 . bis in weite Teile des 19. Jahrhunderts spielt außerdem der sehr prominente essenzialistische Geschlechterdiskurs eine wichtige Rolle. Ausgehend von philosophischen Positionen $^{35}$ werden "Geschlechtercharaktere ${ }^{36}$ angenommen, die Verhaltensweisen, psychologische Dispositionen, körperliche und geistige Fähigkeiten als natürliche Bestandteile des biologischen Geschlechts festschreiben. Aus diesem Geschlechterdiskurs entwickeln sich im Laufe des 19. Jahrhunderts recht mächtige Vorstellungen ,natürlicher Aufgaben- und Raumverteilungen für Mann und Frau im gesellschaftlichen und familiären Gefüge. Das schlägt sowohl auf die Schreibpraxis von Männern und Frauen als auch auf die geschlechtliche Codierung von Autorschaft durch. ${ }^{37}$ Professionelle Autorschaft ist für Frauen nur eingeschränkt möglich, weil sie in der literarischen Öffentlichkeit einmal gegen ihre ,wahre' Aufgabe als Hausfrau ${ }^{38}$ und einmal gegen ihren angeblichen natürlichen ,Geschlechtercharakter' ausgespielt wird. Darüber hinaus erlegt der Literaturdiskurs schreibenden Frauen eine Reihe von ebenso angeblich natürlichen Beschränkungen in Bezug auf Genres, Zielgruppe, Stoffe, Themen und öffentliches Auftreten als professionelle Schreiberinnen auf. ${ }^{39}$ Das bedeutet allerdings nicht, dass sich nicht zahlreiche

34 Vgl. Friedrich A. Kittler, Aufschreibesysteme 1800 · 1900, München: Fink 1995.

35 Johann Gottlieb Fichtes Grundriß des Familienrechts (1797) und die von Wilhelm von Humboldt in Über den Geschlechtsunterschied und dessen Einfluß auf die organische Natur (1794) und Über die männliche und weibliche Form (1795) entwickelte Geschlechteranthropologie sind die bekanntesten Beispiele. Vgl. weiterführend Heidi Ritter, „Wechselseitige Ergänzung: Wilhelm von Humboldts Geschlechteranthropologie zwischen Erfahrung und Konstruktion“, in: Manfred Beetz (Hrsg.), Physis und Norm. Neue Perspektiven der Anthropologie im 18. Jahrhundert, Göttingen: Wallstein 2007, S. 175-186.

36 Vgl. zum essentialistischen Geschlechterdiskurs und den Konzepten von Geschlechtercharakter und Geschlechternatur Helen Fronius, Women and Literature in the Goethe Era 1770-1820. Determined Dilettantes, Oxford: Clarendon Press 2007, S. 10-52, und Barbara Becker-Cantarino, Schriftstellerinnen der Romantik. Epoche - Werk - Wirkung, München: Beck 200o, S. $46 \mathrm{ff}$.

37 Der Zusammenhang zwischen dem essenzialistischen Geschlechterdiskurs und der literarischen Praxis von Männern und Frauen im späten 18. und frühen 19. Jahrhundert findet sich ausführlich illustriert bei Becker-Cantarino, Schriftstellerinnen der Romantik (Anm. 36), S. 53-63, sowie bei Fronius, Women and Literature (Anm. 36), S. 53-93.

38 „Der Gegensatz ,Frau' und ,Autorin‘ im 18. Jahrhundert ist eine Gratwanderung, die zeitgenössischer Literatur zufolge von einigen Auserwählten mit viel Feingefühl bewältigt werden konnte; der Gegensatz ,Hausfrau' und ,Autorin' im 19. repräsentiert einen unüberbrückbaren Abgrund“ (Kord, Sich einen Namen machen [Anm. 31], S. 94).

Vgl. ebd., S. 53 . 
Personen über den dominanten Diskurs hinwegsetzen. Immerhin ist er, wie Fronius betont, in vielen Fällen ein Diskurs, der auf bereits vorhandene Texte und bekannte Frauen reagiert. ${ }^{40}$ Es bedeutet aber, dass wir bei schreibenden Männern und Frauen von sehr unterschiedlichen Ausgangssituationen des Schreibens in Bezug auf Räume, Zeiten, Selbstbild und Veröffentlichungsaussichten ausgehen müssen.

Das gerade grob skizzierte Setting hat unmittelbare Konsequenzen für Fragen nach kollektivem Schreiben in diesem Zeitraum. Dies soll mit Hilfe der Ergebnisse aus dem ersten Teil sowie drei exemplarischen Konstellationen gemischtgeschlechtlicher Zusammenarbeit verfolgt werden - Betsy und Conrad Ferdinand Meyer, Nathaniel und Sophia Hawthorne und Clara und Robert Schumann.

\section{Gemeinsam schreiben und Geschlechtlichkeit im 19. Jahrhundert}

Im ersten Teil hat sich als zentrale Frage erwiesen, welche Handlungen dem Schreibprozess zugerechnet werden und wie sich dies zur Zuweisung von Autorschaft verhält. Interessant ist dazu die jahrzehntelange Zusammenarbeit der Geschwister Meyer, zu der es eine aufschlussreiche Studie von Rosmarie und Hans Zeller gibt. ${ }^{41}$ Die erhaltene Verlagskorrespondenz zeigt, dass Betsy Meyer den größten Teil des Schriftverkehrs und der Verhandlungen für ihren Bruder führt. Darüber hinaus schreibt sie über lange Jahre sämtliche Druckmanuskripte, die an den Verlag gehen, ins Reine - man kann sagen, dass nahezu alle Aufgaben, die den Schreibprozess mit dem Literaturbetrieb verbinden, von ihr durchgeführt werden.$^{42}$ Außerdem ist sie in zahlreiche Vorbereitungsarbeiten involviert - ihre Briefe zeugen davon, dass sie mit ihrem Bruder über geplante Texte diskutiert, Übersetzungen für ihn macht und sich Entwürfe diktieren lässt. Ein Blick in die erhaltenen Manuskripte zeigt außerdem, dass sie seine Entwürfe korrigiert, Formulierungsvorschläge macht und seine Korrekturen wiederum einarbeitet. Ihr Feedback ist dabei durchaus selbstbewusst, auch auf der ästhetischen und sprachlichen Ebene des

40 "It is possible to argue that the theoretical objections against female authorship coexisted with, and were undermined by, practical concessions" (Fronius, Women and Literature [Anm. 36], S. 67).

Vgl. Rosmarie Zeller, „Betsy Meyer, Sekretärin, Kopistin, Mitarbeiterin. Ihre Selbstdarstellung im Briefwechsel mit dem Verleger“, in: Plachta (Hrsg.), Literarische Zusammenarbeit (Anm. 12), S. 157-166, und Hans Zeller, „Betsy Meyers Mitautorschaft an C. F. Meyers Werk“, in: Plachta (Hrsg.), Literarische Zusammenarbeit (Anm. 12), S. 167-196. Vgl. Zeller, „Betsy Meyer“ (Anm. 41). 
Textes - wir finden Randbemerkungen von ihr wie „schwächl[ich]“ oder "nicht klar genug!" ${ }^{43} \mathrm{Am}$ interessantesten sind jedoch die textgenetischen Indizien, die Hans Zeller dafür anführt, dass bestimmte Texte zuerst von ihr konzipiert worden sind. So liegt das Gedicht Der deutsche Schmied in Betsy Meyers Handschrift und in der Erstfassung nachweislich nicht als Diktat vor. ${ }^{44}$ Conrad Ferdinand Meyer ändert an dieser Fassung dann nur noch Interpunktion und Orthographie bzw. wählt aus von ihr vorgeschlagenen Formulierungsvarianten aus. ${ }^{45}$ Nach Howard Beckers These, dass der Kunstbetrieb künstlerische und nicht-künstlerische Tätigkeiten kennt, müssten die Tätigkeiten von Betsy Meyer in diesem Fall eindeutig in die Kategorie der künstlerischen Tätigkeiten fallen. Selbst diese umfassenden und ästhetisch eigenständigen Arbeiten führen jedoch nicht zu einer öffentlichen Anerkennung ihrer Autorschaft - sie tritt bis an ihr Lebensende bei keinem der Texte namentlich in Erscheinung. Um dieses keineswegs seltene Phänomen einer stummen Verfasserschaft zu fassen, lohnt sich ein Blick in zeitgenössische Selbst- und Fremdbeschreibungen gemeinsam arbeitender Frauen und Männer. Es fällt auf, dass Betsy Meyer selbst immer wieder ihre Unselbständigkeit und ihre nachgeordnete Rolle im Schreiben betont. Die Selbstbezeichnungen reichen von "Sekretär ${ }^{446}$ oder sogar „Sekretärchen ${ }^{447}$ zu „Handlanger", wie etwa hier an den Verleger Hermann Haessel: „Durfte ich doch, Sie wissen es ja wohl lieber Haessel, während seines ganzen Schriftstellerlebens sein stiller, bescheidener kritischer Handlanger sein! Er wußte wohl: Selbst machen konnte ich nichts; aber ich verstand ihn. ${ }^{48}$ Hier sehen wir mehrere Schreibgesten, die Susanne Kord als zeittypisch für Frauen bezeichnet hat: zum einen die Hervorhebung der passiven Inspiration und rein praktischen Unterstützung, zum anderen die

43 Gedicht Nr. 206, „Die Seitenwunde“, in: Conrad Ferdinand Meyer, Sämtliche Werke. Historisch-kritische Ausgabe, hrsg. von Hans Zeller und Alfred Zäch, Bd. 5.1, Bern: Benteli 1996, S. 75 .

44 Hans Zeller zeigt ausführlich, wie sich diktierte und selbst konzipierte Entwürfe Betsy Meyers über Indizien wie verwendete Schreibträger und -werkzeuge, Aufzeichnungsformen von Varianten und Sofortkorrekturen unterscheiden lassen (vgl. Zeller, „Betsy Meyers Mitautorschaft" [Anm. 41], S. 172-175).

45 Vgl. Zeller, „Betsy Meyers Mitautorschaft“ (Anm. 41), S. 175 f.

46 Mehrfach in Briefen; eine Auswahl findet sich bei Zeller, „Betsy Meyer“ (Anm. 41), S. 158. Rosmarie Zeller zitiert aus dem unveröffentlichten späten Briefwechsel zwischen Betsy Meyer und Hermann Haessel, der sich in der Conrad Ferdinand Meyer-Sammlung der Zentralbibliothek Zürich befindet und aktuell an der Universität Zürich editorisch erschlossen wird; vgl. http://www.cfmeyer.ch/index.html (letzter Zugriff: 13.08.2020).

47 „Der liebe Bruder ging voran, überarbeitete jeden Morgen von 8-1 Uhr, den vielverzweigten Text, nachmittags schrieb sein altes Sekretärchen den neuen Text ins Reine“ (Brief an Hermann Haessel vom 13.08.1891, zit. n. Zeller, „Betsy Meyer“ [Anm. 41], S. 165). 48 Brief an Hermann Haessel vom 04.09.1894, zit. n. Zeller, „Betsy Meyer“ (Anm. 41), S. 166. 
Betonung der eigenen künstlerischen Minderwertigkeit bzw. „die Beteuerung, die Autorin habe keineswegs versucht, ,Kunst' zu produzieren“.49

Ähnliches sehen wir im Ehetagebuch von Clara und Robert Schumann, wenn sie über gerade fertiggestellte Kompositionen einträgt: „Sind sie freilich von gar keinem Werth, nur ein ganz schwacher Versuch, so rechne ich auf Roberts Nachsicht [...]. Sey gnädig, mein Freund, und schone diese schwache, aber mit voller Liebe gespendete Gabe. ${ }^{50}$ Im weiblichen Schreibverständnis scheint schon die männliche Kritik, sich künstlerische Tätigkeiten und einen künstlerischen Status anzumaßen, den die weibliche Natur eigentlich nicht hergeben dürfte, verinnerlicht und antizipiert. Clara und Robert Schumann sind in diesem Zusammenhang besonders interessant, denn in ihrem gemeinsamen Text tauchen diesbezüglich immer wieder Reibungen auf, wenn etwa Robert Schumann schreibt: „An ihrer Kunst hängt sie begeisterter als je und hat manchmal in der vorigen Woche gespielt, daß ich über die Meisterin die Frau vergaß und sie sehr oft selbst vor anderen geradezu ins Gesicht loben mußte. “51 Die künstlerische Leistung seiner Frau weckt seine Bewunderung und den Impuls, sie öffentlich anzuerkennen - und gleichzeitig seine Verunsicherung, dass er mit dieser Anerkennung vergisst, was sie ist („die Frau“), und dass diese öffentliche Anerkennung „vor anderen“ vielleicht sozial unangemessen sein könnte.

Sowohl die Selbstbeschreibungen Betsy Meyers und Clara Schumanns als auch Robert Schumanns verunsicherter Kommentar zeugen davon, dass das Arbeiten auf Augenhöhe und dessen Öffentlichkeit Gegenstand der Verhandlung sind. Komplementär zur Herabsetzung der weiblichen künstlerischen Arbeit wird die des Mannes in allen Fällen überhöht und mit Attributen des Genialen ausgestattet:

[E]s schmerzt mich aufs tiefste, wenn ich Dich um Geld bitten muß, und Du mir Dein Erworbenes giebst, es ist mir oft, als müßte dies alle Poesie von Deinem Leben rauben. Du bist so ein Künstler im ächten Sinne des Wort's, Dein ganzes Dichten und Trachten hat mir so etwas zartes, poetisches, ich möchte sagen heiliges, daß ich Dich gern mit aller Prosa, wie sie nun doch einmal im ehelichen Leben nicht ausbleibt, verschonen möchte, es verwundet mich, Dich so gar oft aus Deinen schönen Träumen reißen zu müssen, ${ }^{52}$

49 Kord, Sich einen Namen machen (Anm. 31). S. 64.

$50 \quad$ Robert Schumann und Clara Schumann, Ehetagebücher, hrsg. von Gerd Nauhaus und Ingrid Bodsch, Frankfurt/Main: Stroemfeld 2013, S. 49 f.

51 Ebd., S. 29.

$5^{2}$ Ebd., S. 155 . 
schreibt Clara Schumann. Ähnlich klingt Sophia Hawthornes Beobachtung ihres Mannes bei der Gartenarbeit:

Liebster Gatte, du solltest nicht arbeiten müssen, vor allem nicht mit den Händen, \& du hasst es zu Recht. Du bist ein Engel, der kam, die noch schlafende Natur \& die Menschen zu beobachten, ohne dazu gezwungen zu sein, dich mit Fortpflanzung oder dem Wegräumen von altem Abfall abzumühen. Apollo inmitten seiner Herden hätte nicht so deplatziert aussehen können wie du mit Säge \& Axt \& Rechen in der Hand. ${ }^{53}$

In beiden Fällen wird dem Mann eine überirdische Befähigung zu künstlerischen Handlungen zugesprochen, die gegen alltägliche, nicht-künstlerische Aufgaben ausgespielt wird. Ähnliches gibt es auch bei Betsy Meyer, die beim Verleger um Entschuldigung dafür bittet, dass sie den Antwortbrief schreibt, da ihr Bruder nicht beim Schreiben gestört werden dürfe. ${ }^{54}$ Wir sehen bei den männlichen Schreibenden eine strikte Trennung von künstlerischer Tätigkeit und privater, alltäglicher Tätigkeit, die die schreibenden Frauen für ihr eigenes Schreiben nicht ziehen. Mehr noch: Bei den Männern sind die nichtkünstlerischen Alltagsaufgaben das Hindernis für ihre wahre Bestimmung, bei den Frauen die künstlerischen Aufgaben. Dieser Unterschied ist in Bezug auf männliche und weibliche Autorschaft dieser Zeit und speziell in Bezug auf kollektives Schreiben von großer Bedeutung. Männer, die schreiben oder anders künstlerisch tätig sind, üben darin ihren Beruf und ihre vermeintlich ,natürliche` Bestimmung aus, während Frauen, die schreiben, in Konkurrenz treten zu ihrem häuslichen ,Beruf':

Klara hat eine Reihe von kleineren Stücken geschrieben, in der die Erfindung so zart und musikreich, wie's ihr früher noch nicht gelungen. Aber Kinder haben und einen immer phantasirenden Mann, und componiren geht nicht zusammen. Es fehlt ihr die anhaltende Übung, und dies rührt mich oft, da so mancher innige Gedanke verloren geht, den sie nicht auszuführen vermag. Klara kennt aber selbst ihren Hauptberuf als Mutter, daß ich glaube, sie ist glücklich in den Verhältnissen, wie sie sich nun einmal nicht ändern lassen. ${ }^{55}$

Robert Schumann erkennt an, dass seine Frau "so manche[] innige Gedanke[n]" künstlerischer Art hat, und bedauert, dass diese nicht zur Ausführung kommen. Doch auch er kann ihre häuslichen Pflichten nur als ihren

53 Sophia Hawthorne und Nathaniel Hawthorne, Das Paradies der kleinen Dinge. Ein gemeinsames Tagebuch, hrsg. und übers. von Alexander Pechmann, Salzburg: Jung und Jung 2014, S. 131 (Herv. im Orig.).

54 Vgl. Zeller, „Betsy Meyer“ (Anm. 41), S. 160.

55 Schumann/Schumann, Ehetagebücher (Anm. 50), S. 159. 
"Hauptberuf" denken und als naturgegeben für sie bestimmt hinnehmen. Dabei spielt es übrigens keine Rolle, dass speziell Clara Schumann als Konzertpianistin über Jahre substanziell zum Familieneinkommen beiträgt und in der Öffentlichkeit durchaus als eigenständige Künstlerin wahrgenommen wird. Die künstlerische Praxis und ihre Repräsentation liegen oft auseinander - das zeigt auch das Beispiel von Betsy Meyer.

Was lässt sich bis hierher über geschlechtliche Implikationen des kollektiven Schreibens sagen? Der betrachtete Zeitraum ist von stark geschlechtsgebundenen Selbstbildern im Schreiben und Rhetoriken über das eigene und fremde Schreiben geprägt. ${ }^{56}$ Weiterhin zeigt sich, dass die räumliche und zeitliche Grundlage männlicher und weiblicher Schreibszenen sehr unterschiedlich ist. In allen drei Beispielen werden die künstlerischen Tätigkeiten zwar durchaus auch von den Frauen ausgeübt und von den männlichen Mitschreibern anerkannt (auch Conrad Ferdinand Meyers unveränderte Übernahme des Gedichts seiner Schwester ist eine Art der Anerkennung). Nathaniel Hawthorne räumt sogar ein, dass seine Frau über bestimmte Gegenstände besser schreibe als er: „An schönen Tagen ist das Hauptereignis und die glücklichste Stunde des Nachmittags ein Spaziergang mit meiner Frau. Sie muss diese Spaziergänge beschreiben, denn wann immer sie und ich etwas gemeinsam genossen haben, hielt ich meine Feder stets für unwürdig und unangemessen, es zu notieren. “57 Trotzdem steht der öffentliche Status als Künstlerin bei allen drei Frauen mehr oder weniger stark ausgeprägt in Frage, während er bei allen drei Männern unhinterfragt ist oder sogar kultiviert wird.

\section{Kollektives Schreiben und Geschlechtlichkeit - ein (vorläufiges) Fazit}

Blickt man zum Abschluss noch einmal auf die eingangs formulierte (und keinesfalls vollständige) Aspektsammlung zu kollektiven Schreibprozessen, so sind die geschlechtlichen Implikationen dieser Aspekte an den Beispielen deutlich geworden: Die Frage nach den Beteiligten am Schreibprozess involviert zugleich jene danach, wer vom Schreibprozess von Geschlechts wegen ausgeschlossen wird bzw. sich selbst ausschließt. Noch stärker vom Faktor Geschlecht bedingt ist die öffentliche, namentliche Repräsentation eines

\footnotetext{
56 Hierzu passt auch Susanne Kords Beobachtung, dass sich viele schreibende Frauen im relevanten Zeitraum für betont harmlose und naive Pseudonyme entscheiden (vgl. Kord, Sich einen Namen machen [Anm. 31], S. 14).

57 Hawthorne/Hawthorne, Das Paradies der kleinen Dinge (Anm. 53), S. 5 o.
} 
Schreibprozesses. Auch die Aspekte der Organisation des Schreibprozesses und des Selbst- und Fremdbilds der Schreibenden lassen sich erweitern um die Fragen, inwiefern bestimmte Handlungen innerhalb von Schreibprozessen geschlechtlich codiert sind (etwa das Korrigieren der Texte oder das Korrespondieren nach Außen versus den Entwurf einer ersten Textfassung auf dem leeren Blatt) und was das für den Stellenwert dieser Handlungen in der öffentlichen Repräsentation bedeutet. Mit dem 19. Jahrhundert wurde zugegebenermaßen ein Zeitraum betrachtet, in dem das Geschlecht ein besonders großer Konfliktfaktor im Schreiben ist. Folgt man aber der Annahme, dass die bis heute dominante Vorstellung ${ }^{58}$ einer singulären Autorschaft in dieser Zeit maßgeblich ausgeprägt und affirmiert wird, lassen sich daraus wertvolle Einsichten für die Erforschung kollektiven Schreibens gewinnen.

Ich bin eingangs davon ausgegangen, dass weibliches Schreiben und kollektives Schreiben Gemeinsamkeiten besitzen. Wie in den 1970er Jahren durch die systematische Erforschung weiblicher Autorschaft zahlreiche Texte jenseits des Kanons in den Blick kommen, richtet auch das Interesse an kollektivem Schreiben den Fokus sowohl auf bislang unbeachtetes Textmaterial (wie etwa im privaten Rahmen geführte gemeinsame Tage- und Notizbücher) als auch auf bislang unbeleuchtete Teile von bereits bekannten Texten und Schreibprozessen (wie die stummen Mitverfasserschaften an kanonisierten Gedichten, die Stillinger und Zeller herausarbeiten). Ein Schlüsselelement dieser Fokuserweiterung scheint sowohl für das weibliche als auch für das kollektive Schreiben die Öffnung des Schreibprozesses in den privaten Raum zu sein. Es ist die aus dem 19. Jahrhundert herrührende Dichotomie zwischen einem beruflichen Schreibraum, in dem die künstlerischen Tätigkeiten passieren und der zur Grundlage von Autorschaft wird, und einem privaten Raum, in dem die vermeintlichen Nebenschauplätze angesiedelt sind, die, wie schon die wenigen Beispiele gezeigt haben, den Blick sowohl auf weibliches Schreiben als auch auf viele kollektive Elemente des Schreibprozesses verstellen.

Christa Bürger hat für das Schreiben von Frauen im 19. Jahrhundert dargelegt, dass deren historische Schreibpraxis nur über eine enge konzeptionelle Verschränkung von sozialem Leben und Schreibakt angemessen beschreibbar

$5^{8}$ Auch der Paradigmenwechsel im Rahmen des Poststrukturalismus, den die einschlägigen Beiträge von Roland Barthes (Der Tod des Autors, 1967) und Michel Foucault (Was ist ein Autor?, 1969) für die Autorschaftsforschung in mehrerer Hinsicht bedeuten, lässt das Konzept der singulären Autorschaft weitgehend unberührt. 
ist. ${ }^{59}$ Vor allem für die unmittelbar kollaborativen Konstellationen lässt sich Ähnliches sagen - sie sind kaum in den Blick zu bekommen, wenn die Perspektive auf den Schreibprozess abgekoppelt wird von anderen Geschehnissen des sozialen Lebens.

Was bedeutet das - in aller Kürze - für die literaturwissenschaftliche Arbeit an kollektivem Schreiben? Was Susanne Kord über die Literatur von Frauen sagt, ${ }^{60}$ gilt auch hier: Gerade bislang marginalisierte Texte entziehen sich unter Umständen den Werkzeugen, Bezügen und Strukturen, die am männlichen Kanon bzw. dem der singulären Autorschaft geschult sind. Sie mit diesen Maßstäben zu messen, führt häufig dazu, sie als unbedeutend abzutun. Eine Forschung zu kollektivem Schreiben muss bereit sein, an ihm gegebenenfalls Werkzeuge und literaturgeschichtliche Strukturen neu auszurichten. Das kann beispielsweise bedeuten, Texte und Tätigkeiten jenseits des öffentlichen Raums wahrzunehmen. Es kann erfordern, Dichotomien wie privat/öffentlich, Professionelle/Amateure, Leben/Schreiben, künstlerische/ nicht-künstlerische Tätigkeit in Bezug auf Autorschaft aufzuweichen. Es setzt voraus, die öffentliche Darstellung eines Schreibprozesses nicht als sein Abbild zu betrachten, sondern ihre von Campe und Stingelin attestierte szenische Qualität ernst zu nehmen und sie als Inszenierung ausgewählter Anteile und Personen zu betrachten. Es kann bedeuten, auf bestimmte Fragen im ersten Schritt zu verzichten, zum Beispiel auf die nach dem ,wahren Autor' oder der ,wahren Autorin' eines Textes oder nach der künstlerischen Wertigkeit einer Tätigkeit im Rahmen eines Schreibprozesses.

Die Untersuchungen von Hans und Rosmarie Zeller zu Conrad Ferdinand und Betsy Meyer zeigen in ihrer Materialbasis, wie ein produktiver Zugang zu gemeinsamen Schreibprozessen aussehen kann: Hier wird mit Manuskriptmaterial und textgenetischen Methoden ebenso gearbeitet wie mit SchreibSzenen in überlieferter Korrespondenz und autobiografischen Dokumenten.

59 Vgl. Christa Bürger, Leben Schreiben. Die Klassik, die Romantik und der Ort der Frauen, Stuttgart: Metzler 199o.

6o „Daß wir nicht gelernt haben (und bis heute in traditionellen Kurrikula nicht lernen), die Literatur von Frauen mit Genuß zu lesen, liegt an zwei Tatsachen: erstens unterscheidet sich viele (nicht alle) Literatur von Frauen erheblich von männlicher Literatur, formell und inhaltlich, und zweitens beschränkt sich unser Wissen und Training auf männliche Literatur und deren Formen und Inhalte. Die Literatur von Männern wird heute noch an den meisten Universitäten unter dem Namen ,Weltliteratur' gelehrt; unser literarisches Wissen besteht größtenteils darin, die Formen und Inhalte männlicher Literatur zu erkennen, anzuerkennen, zu analysieren, zu kritisieren, zu reflektieren, Bezüge zur männlichen Literaturgeschichte herzustellen usw." (Kord, Sich einen Namen machen [Anm. 31], S. 21). 
Denn erst diese Vielseitigkeit des Zugriffs zeigt, dass Betsy Meyers Selbstbild als Schreiberin, das sie im Rahmen diverser Schreib-Szenen entwirft, nicht mit dem Manuskriptmaterial übereinstimmt. Und auch ganz generell wird man in kollektiven Schreibprozessen darauf vorbereitet sein müssen, dass die Stimmen der unterschiedlichen Akteurinnen und Akteure und die Materialspuren häufig eine unterschiedliche Sprache sprechen. 